\title{
Probing the 2-D kinematic structure of early-type galaxies out to 3 effective radii
}

\author{
Robert N. Proctor ${ }^{1}$, Duncan A. Forbes ${ }^{1}$, Aaron J. Romanowsky ${ }^{2}$, \\ Jean P. Brodie ${ }^{2}$, Jay Strader ${ }^{3}$, Max Spolaor ${ }^{1}$, J. Trevor Mendel ${ }^{1}$ \\ and Lee Spitler ${ }^{1}$ \\ ${ }^{1}$ Centre for Astrophysics \& Supercomputing, Swinburne University, Hawthorn VIC 3122, \\ Australia \\ ${ }^{2}$ UCO/Lick Observatory, University of California, Santa Cruz, CA 95064, USA \\ ${ }^{3}$ Harvard-Smithsonian Centre for Astrophysics, 60 Garden St., Cambridge, MA 02138, USA \\ email: rproctor@astro.iag.usp.br
}

We detail an innovative new technique for measuring the 2-D velocity moments (rotation velocity, velocity dispersion and Gauss-Hermite coefficients $h_{3}$ and $h_{4}$ ) using spectra from Keck DEIMOS multi-object spectroscopic observations. The data are used to reconstruct $2-\mathrm{D}$ rotation velocity maps.

Here we present data for two nearby early-type galaxies to $\sim 3$ effective radii. From these data 2D kinematic maps are constructed. Three other galaxies have been similarly analysed (see 2009, MNRAS, 398, 91). We provide significant insights into the global kinematic structure of these galaxies, and, in some cases, challenge the accepted morphological classification. We show that between 1-3 effective radii the velocity dispersion declines very slowly, if at all, in all five galaxies. For the two galaxies with velocity dispersion profiles available from planetary nebulae data we find very good agreement with our stellar profiles. We find a variety of rotation profiles beyond 1 effective radius, i.e remain constant, decrease and increase with radius. These results are of particular importance to studies which attempt to classify galaxies by their kinematic structure within one effective radius, such as the recent definition of fast- and slow- rotator classes by the SAURON project. Our data suggests that the rotator class may change when larger galacto-centric radii are probed. This has important implications for dynamical modelling of early-type galaxies. The data from this study are available on-line. 